\title{
LA EFICACIA DE LA ESCAPE ROOM COMO ESTRATEGIA DE MOTIVACIÓN, COHESIÓN Y APRENDIZAJE DE MATEMÁTICAS EN SEXTO DE EDUCACIÓN PRIMARIA
}

\author{
THE EFFECTIVENESS OF THE ESCAPE ROOM AS A STRATEGY \\ OF MOTIVATION, COHESION AND LEARNING OF MATHEMATICS \\ IN SIXTH OF PRIMARY EDUCATION
}

\section{Nuria Zarco Claudio ${ }^{a}$ Mónica Machancoses ${ }^{b}$ Rocío Fernández Piqueras ${ }^{c^{*}}$}

Fechas de recepción y aceptación: 24 de octubre de 2019, 8 de diciembre de 2019

Resumen: El presente trabajo de investigación es un caso práctico, analizado desde la realización de una experiencia innovadora en el aula utilizando recursos TIC y metodologías activas de aprendizaje. Concretamente, se trata de analizar los múltiples beneficios que la nueva tendencia de los escape rooms brinda al mundo de la educación. En esta línea, observaremos las aportaciones de diferentes autores y autoras que defienden la inclusión de metodologías innovadoras en el aula con el fin de vincular el aprendizaje con la motivación y la diversión. Para lograrlo, procederemos al diseño de una experiencia didáctica de escape room titulada "la extraña desaparición de Don José". Esta se enmarca en un contexto educativo concreto y se camufla bajo la pretensión de repasar contenidos matemáticos de sexto curso de educación primaria. Además, se analizarán a través de la experiencia las habilidades sociales y emocionales de los estudiantes, así como el desarrollo de su competencia digital y grado de cohesión grupal.

Palabras clave: escape room educativo, innovación TIC.

${ }^{a}$ Maestra en Educación Primaria.

${ }^{b}$ Directora de Infantil. Coordinadora TIC del Colegio Internacional Ausiàs March (Picassent), Valencia).

c Departamento de Didáctica General, Teoría de la Educación e Innovación Educativa. Facultad de Magisterio y Ciencias de la Educación. Universidad Católica de Valencia San Vicente Mártir.

* Correspondencia: Universidad Católica de Valencia San Vicente Mártir. Facultad de Magisterio y Ciencias de la Educación. Calle Sagrado Corazón, 5. 46110 (Godella), Valencia. España.

E-mail: rocio@ucv.es 
Abstract: This research work is a practical case, analyzed from the realization of an innovative experience in the classroom using ICT resources and active learning methodologies. More specifically, it analyzes the multiple benefits that the new trend of escape rooms mean in the educational field. In this line, we will observe the contributions of different authors who defend the inclusion of innovative methodologies in the classroom in order to link learning with motivation and fun. To achieve this, we will proceed to the design of a didactic escape room experience entitled "the strange disappearance of Don José". This is framed in a specific educational context and is camouflaged with the intention of reviewing mathematical contents in the sixth year of primary education. In addition, the social and emotional skills of the students will be also analyzed through experience, as well as the development of their digital competence and the degree of the group cohesion.

Keywords: Educational escape room, ICT innovation.

\section{INTRODUCCIÓN}

Hoy en día el docente dispone de múltiples herramientas tecnológicas, en mayor o menor medida útiles para su quehacer diario. Además, gracias a internet y las diferentes redes sociales es posible conocer experiencias didácticas y testimonios que otros docentes innovadores llevan a cabo en sus aulas. Por otra parte, el sistema educativo nos habla de un cambio de enfoque en relación con las competencias y objetivos que hay que lograr en los alumnos; necesitamos que los docentes desarrollen habilidades que vayan a aplicar de una forma práctica en la vida real, por ejemplo, competencias de liderazgo, democracia, educación emocional y trabajo en equipo, frente a la memorización y repetición (Área, 2008).

Parece claro establecer unos mecanismos de mejora en los procesos de enseñanza y de aprendizaje, pero pocas veces se reflexiona sobre ello; es decir, ¿por qué hay metodologías que sí funcionan y otras no?

Estos mecanismos de mejora vienen de la mano de nuevas estrategias y metodologías que defienden la importancia de motivar a los alumnos para lograr un aprendizaje significativo y efectivo (Naranjo, 2009). Esto está en consonancia con la mayoría de investigaciones realizadas en las aulas, que apuntan a que la motivación es un factor determinante en la conducta de los estudiantes y por ende en su proceso de aprendizaje. Podría ser este un motivo de peso para que los maestros y maestras investiguen, conozcan y trabajen en 
todos aquellos condicionantes que favorecen la motivación tanto extrínseca como intrínseca de los estudiantes de hoy en día. Ahora bien, ¿qué necesitan los alumnos para estar motivados?

Estas cuestiones y otras muchas son las que nos planteamos a diario todos aquellos/as que dedicamos nuestra vida a la enseñanza y que no dejamos de investigar persiguiendo una mejora de nuestra acción docente y, por ende, del sistema educativo. Esta investigación-acción llevada al aula de primaria se va a centrar en torno al análisis de la eficacia de una de las metodologías activas de aprendizaje basado en juego que están teniendo más éxito recientemente, como es el escape room educativo.

\section{ESCAPE ROOM: SU CONCEPTO Y ORIGEN}

Si indagamos sobre su origen, aparece el nombre de Takao Kato, guionista y director de cine japonés que ideó en 2008 el Real Escape Game (REG), es decir, el primer juego de aventura físico y mental que consistía principalmente en resolver diferentes enigmas entre un grupo de jugadores. En pocos años, su auge se extendió por Asia, California, Europa y Australia, hasta ser en 2013 cuando se estrenan en España, concretamente en Barcelona. Actualmente, esta tendencia de ocio ha dado la vuelta al mundo con el nombre de escape room, $\mathrm{y}$ tal ha sido su acogida por parte de la sociedad que se calculan más de 350 empresas de juegos de escapismo en toda España, convirtiendo a los españoles en los mayores usuarios de toda Europa (Moya, 2019).

En este caso, en el contexto educativo adquiere el nombre de habitaciones de escape educativas o educational escape rooms, y se justifican como un sistema de juego dentro de la escuela. En este sentido, según el contexto en el que se lleve a cabo la experiencia, se puede vincular esta práctica educativa dentro de diferentes metodologías como, por ejemplo, el aprendizaje basado en juegos (ABJ) o game based learning (GBL), juego serio o serious game o, finalmente, gamificación (García, 2018).

Para que la escape room resulte eficaz y útil para el aprendizaje de los estudiantes, se debe tener en cuenta que se trata de un juego en el que intervienen múltiples variantes que han de ser consideradas en función del grupo de alumnos que se disponga (agrupaciones, tiempo, dificultad, objetivos de 
aprendizaje, tema y espacio, tecnología y materiales, evaluación, etc.). A todo ello, Cordero (2018) añade que no se debe perder de vista que el éxito y la eficacia de este juego se encuentra en el choque cognitivo inicial y el conflicto que se produce en la mente del alumnado, ya que este elemento es el principal causante de la inmersión de los participantes en el juego. Por tanto, queda manifiesta la importancia de diseñar un arranque inicial del juego que sea atractivo y novedoso para los participantes, de modo que logre captar su atención y despertar su motivación.

\subsection{Elementos que componen una experiencia de escape room}

Segura y Parra (2019) resaltan la importancia de los siguientes:

- Tiempo: no hay un tiempo estándar porque puede haber variaciones según cada experiencia de escape room; no obstante, sí es conveniente fraccionarlo en tres momentos diferenciados del juego teniendo en cuenta las acciones que se debe realizar por parte del game master antes, durante y después del juego.

- Dificultad: se debe buscar un equilibrio en la dificultad de las actividades y retos propuestos, de modo que no resulten ni muy fáciles ni demasiado complicados y se adapten al tiempo nivel de los jugadores.

- Objetivos de aprendizaje: en el caso de las habitaciones de escape educativas, se debe establecer unos objetivos previamente, centrar la experiencia en torno a ellos y posteriormente evaluarlos.

- Tema y espacio: se debe adaptar al máximo posible el espacio físico y el tema o narrativa que conduce la historia del juego para lograr la motivación de los participantes.

- Enigmas: se trata de la parte central del juego y debe vertebrar todo el diseño de la experiencia; por tanto hay que intentar que sea atractivo y creativo para los jugadores.

- Tecnología y materiales: las nuevas tecnologías aplicadas en la educación pueden mejorar la experiencia a través de su uso por parte del game master en el diseño de los enigmas y por parte de los alumnos para la resolución de estos. 
- Evaluación: permitirá obtener información en torno al progreso de los alumnos a lo largo del escape room, la dificultad, el tiempo de resolución y el nivel de cooperación que se ha despertado entre ellos.

- Ensayo: se debe realizar siempre al menos una vez antes de iniciar el juego, en ausencia de los participantes, para comprobar que todo está en el orden adecuado y funciona como se espera.

Todos estos elementos deben ser necesariamente valorados si se pretende diseñar una propuesta de escape room que resulte eficaz y útil para el aprendizaje de los estudiantes, a lo que Cordero (2018) añade que no se debe perder de vista que el éxito y la eficacia de este juego se encuentran en el choque cognitivo inicial y el conflicto que se produce en la mente del alumnado, ya que este elemento es el principal causante de la inmersión de los participantes en el juego. De acuerdo con ello, queda manifiesta la importancia de diseñar un arranque inicial del juego atractivo y novedoso para los participantes, de modo que logre captar su atención y despertar su motivación por él.

\subsection{Beneficios de la escape room en el aula}

De acuerdo con Martínez, Poyatos y Fernández (2018), llevando el escape room a las aulas, favorecemos las siguientes conexiones pedagógicas:

- Aprendizaje basado en retos.

- Gamificación.

- Trabajo cooperativo.

- M-learning.

- Inteligencias múltiples.

- Conexión curricular.

Del mismo modo, Segura y Parra (2019) coinciden en la utilidad del escape room para enseñar valiosas habilidades que pueden ser utilizadas a nivel educativo y además podrían resultar muy útiles para el futuro de los estudiantes; entre ellas destaca el respeto, la gestión del tiempo, el trabajo bajo presión, la resolución de problemas y sobre todo la comunicación. 
Además, cabe destacar que dicha interdependencia se genera a partir de habilidades que son elementales para ellos, como son la comprensión, la escucha a los demás compañeros o la capacidad de decisión frente a los enigmas. También suscita valores muy perseguidos en el campo de la educación, como el respeto por la diversidad, la tolerancia frente a opiniones diferentes, la responsabilidad de nuestras actuaciones y la equidad (García, 2018).

Sin embargo, lejos de quedar aquí, el aprendizaje en grupo lleva implícito otro de los grandes aprendizajes que se dan dentro del terreno educativo y que comúnmente responde al nombre de "aprendizaje entre iguales". En consonancia con las aportaciones de Vygostky (1978), y extrapolándolas al juego de escape room, comprendemos que los alumnos interactúan entre ellos y socializan poniendo su conocimiento al servicio de los demás compañeros, de modo que todos aportan sus cualidades al grupo buscando un bien común (Gil-Quintana y Ortega, 2018).

Como vemos, esta vivencia didáctica puede enriquecer a los estudiantes en diversos ámbitos (educativo, intelectual, social, psicológico, emocional y físico). Todos ellos útiles tanto dentro como fuera del aula, de modo que conviene poner sobre una balanza aquellos puntos que nos impiden animarnos a llevarlo a cabo y sopesarlo junto a este manojo de beneficios, de modo que podamos observar si realmente merece la pena arriesgarse o no.

\section{MÉTODO}

La presente investigación nace dentro del trabajo de fin de máster en Innovación Tecnológica en Educación de la Universidad Católica de Valencia y, por tanto, es coordinada desde la Universidad, para ser realizada y analizada desde un centro educativo. El objetivo general que se plantea es que a partir de una experiencia innovadora en el aula se analice su efecto posterior de mejora en el proceso de enseñanza y aprendizaje. Concretamente, se analizará la eficacia de una experiencia de escape room como estrategia de aprendizaje en la asignatura de matemáticas de sexto de primaria.

A partir de ahí, se plantean varias hipótesis iniciales sobre los efectos que la innovación podría despertar en el aula: 
La aplicación de escape room permite...:

H.1. Incrementar la motivación del alumnado.

H.2. Trabajar todas las competencias clave de forma integrada.

H.3. Generar un aprendizaje activo y basado en el descubrimiento.

H.4. Potenciar la ayuda entre iguales.

H.5. Desarrollar estrategias útiles para la resolución de conflictos.

H.6. Observar las actitudes, caracteres y roles del alumnado.

La metodología seleccionada para la presente investigación está basada en el método acción-investigación desarrollado por varios autores, como Carr y Kemmis (1986). Con este método se defiende la necesidad de comprender la enseñanza como una acción práctica de carácter social, la cual puede ser mejorada a través de proyectos e investigaciones que pasen por las fases de planificación, acción, observación y reflexión. En línea con esta idea, el presente trabajo confía en el escape room como un elemento innovador que podría mejorar la práctica docente y en consecuencia favorecer el aprendizaje del alumnado. Por tanto, se pretende diseñar una escape room para ponerla a prueba en un aula de Educación Primaria, analizar posteriormente los resultados obtenidos y de este modo poder reflexionar sobre la práctica realizada (Latorre, 2003).

De acuerdo con todo ello, se combinarán instrumentos de recogida de datos tanto cualitativos como cuantitativos que permitirán recoger información de la experiencia y en torno a los cuales se podrá reflexionar. Se diseñó un cuestionario propio, sencillo, de diez preguntas, destinado a que los niños contestaran su opinión una vez finalizada la experiencia. El cuestionario se diseñó también con la herramienta de Google Form y se utilizó una escala likert sencilla donde 1 era el peor valor y 4 el máximo para valorar las afirmaciones anónimas de los estudiantes. Se utilizó para la observación participante un tabla a modo de registro de observación (construida a partir de las directrices de Gutiérrez, 2018) para marcar los ítems más relevantes para el estudio durante la realización de la experiencia. Por último se empleó como instrumento cualitativo una entrevista cerrada y estructurada de 6 preguntas, que se realizaría posteriormente al docente tutor del aula, que también participaba en la experiencia (Mayorga, 2004). 


\section{DESCRIPCIÓN DE LA EXPERIENCIA}

\subsection{La muestra: el centro y el alumnado}

La coyuntura en la que se enmarca la presente investigación es en el Colegio Internacional Ausiàs March, del municipio de Picassent (Valencia). Se trata de un centro privado, desde el cual se apuesta por la innovación educativa y se fomenta el uso de las nuevas tecnologías aplicadas a la educación en todas sus etapas educativas.

Concretamente, el diseño de la presente propuesta va dirigido al grupo de alumnos de sexto de primaria, 25 alumnos/as con elevado nivel cognitivo y manejo de las nuevas tecnologías, puesto que las emplean de forma cotidiana en el aula en diferentes asignaturas. Sin embargo, en el grupo se detectan algunas dificultades en cuanto a la cohesión y cooperación de este, siendo reincidentes discusiones derivadas de proyectos grupales que han culminado en el rechazo por parte de algunos alumnos/as hacia este tipo de actividades, incluso hacia el trabajo con determinados compañeros, teniendo como preferencia los trabajos individuales. De este modo, podríamos decir que en el aula se respira cierto clima de competitividad al que deberá hacer frente la propuesta de la escape room.

\subsection{Diseño de la innovación: la escape room}

La escape room diseñada se ubicó en la modalidad de "escape room a gran escala" y los enigmas que había que resolver siguieron una ruta lineal. Se enmarca en el contexto de la asignatura de matemáticas de sexto curso y el contenido principal que trabajar son las áreas de polígonos regulares.

En cuanto a los objetivos específicos de la propuesta, se plantearon algunos de carácter intelectual, social, emocional y psicológico, todos ellos relacionados con las competencias clave del currículum de Primaria, como se indica en la tabla 1. 
TABLA 1

Objetivos de la propuesta de escape room

\begin{tabular}{|c|c|c|}
\hline Objetivos de la propuesta & Carácter & Comp. Clave \\
\hline $\begin{array}{l}\text { a) Desarrollar la competencia matemática en todas sus vertientes, a } \\
\text { través de coordenadas, fórmulas matemáticas, puzles, ejercicios de } \\
\text { lógica, mediciones y problemas. }\end{array}$ & Intelectual & CMCT \\
\hline b) Repasar y consolidar las áreas de los polígonos regulares. & Intelectual & CMCT \\
\hline $\begin{array}{l}\text { c) Desarrollar la competencia digital a través del manejo de Google } \\
\text { Form, Youtube, correo electrónico, blogs, códigos QR, imágenes } \\
\text { digitales y audios. }\end{array}$ & Intelectual & $\mathrm{CD}$ \\
\hline $\begin{array}{l}\text { d) Trabajar la competencia en comunicación lingüística a través de la } \\
\text { comprensión de los enigmas y en la comunicación con el equipo en la } \\
\text { toma de decisiones. }\end{array}$ & Social & CCLI \\
\hline e) Aprender a gestionar el tiempo y los recursos disponibles. & Intelectual & CAA \\
\hline f) Cooperar y coordinarse en equipo para resolver los enigmas. & Social & SIEE \\
\hline $\begin{array}{l}\text { g) Favorecer la ayuda entre iguales y la mejora de las relaciones so- } \\
\text { ciales. }\end{array}$ & Social & $\mathrm{CSC}$ \\
\hline $\begin{array}{l}\text { h) Gestionar las emociones positivas y negativas suscitadas durante la } \\
\text { actividad. }\end{array}$ & Emocional & CAA \\
\hline i) Evadirse de la rutina y aprender divirtiéndose con la actividad. & Psicológico & CAA \\
\hline
\end{tabular}

Una vez establecidos los objetivos, se diseñaron las actividades matemáticas que había que resolver, es decir, los "enigmas". El alumnado tenía que resolver siete enigmas con cinco variaciones en cada uno de ellos, una para cada grupo. Los enigmas planteados fueron:

- Enigma 1: Coordenadas de una figura: Sobre un eje de coordenadas, unir unos puntos de coordenadas concretos para construir una figura regular y calcular su área.

- Enigma 2: Fórmulas matemáticas: Los alumnos/as deben encontrar una fórmula conocida, ya estudiada, para calcular el área de las figuras planas, pero en lugar de los números habrá letras. Deberán reconocer de qué formula se trata y sustituir las letras por la posición que ocupa en el 
abecedario para dar con el número que corresponde y poder calcular el área a través de la fórmula.

- Enigma 3: Cálculo del área de una parte de la figura: A partir de una imagen creada digitalmente y compuesta por diferentes figuras geométricas de colores, el alumnado deberá calcular el área de una parte de la figura concreta. Para ello, solamente dispondrá de las medidas de ancho y largo de la figura global, y deberá llevar a cabo un ejercicio de observación y emplear la lógica para determinar las medidas de los lados de su figura y poder calcular su área.

- Enigma 4: Problema matemático: Comprensión del enunciado de un problema que deriva en el cálculo del área de una figura plana.

- Enigma 5: Actividad de medición en el entorno: Con ayuda de la cinta métrica, el alumnado deberá obtener las medidas reales de un objeto del aula y calcular su área.

- Enigma 6: Construcción de una figura a través del tangram: Los alumnos/as deberán unir las piezas de un tangram de cartón, elaborado para la ocasión, para posteriormente calcular el área de la figura resultante.

- Enigma 7: Código morse. Partiendo de un audio, deberán escuchar con atención la secuencia de pitidos cortos y largos, anotarla, y posteriormente sustituirla por las letras que corresponden para descifrar el mensaje.

La narrativa de la escape room, elemento fundamental dentro del juego, consistió en una hipotética investigación por parte de la guardia civil para resolver un caso de desaparición de un docente del colegio, Don José.

El juego comenzaba con un breve vídeo (creado expresamente), a través del cual se proyecta una noticia del telediario, "La desaparición de un profesor en el colegio Ausiàs March de Picassent". Al final del vídeo, se transmite calma y seguridad a la ciudadanía dando a entender que el caso se encuentra en las mejores manos, puesto que existen cinco brigadas de la guardia civil, cuyos agentes han sido seleccionados de manera minuciosa, trabajando en el caso. Además, se comunica que las cinco brigadas de agentes peinarán el aula del docente próximamente en busca de cualquier prueba relevante para la investigación. De este modo, de una forma breve, sencilla y atractiva, quedó explicada la trama, repartidos los papeles de los personajes, y establecido el 
escenario del juego. Ahora bien, el juego se diseñó de forma que solamente podían resolverlo si los cinco grupos llegaban hasta el final, por lo que se intentó fomentar no una competitividad entre los equipos, sino una colaboración y ayuda entre iguales. El juego finaliza cuando cada grupo consiga un símbolo al final del recorrido, el cual se corresponde con una letra o número que deberán traducir y que corresponde con una de las letras o números que son necesarios para desbloquear el ordenador de Don José, el docente desaparecido, conociendo así los motivos de su desaparición.

Continuando con la narrativa, para dotar de significado los enigmas matemáticos, se diseñaron las pistas para guiar al alumnado al lugar dónde se encuentra cada enigma (tabla 2).

TABLA 2

Pistas del lugar y acceso a los enigmas

\begin{tabular}{|c|c|c|}
\hline Enigma & Pista del lugar & Acceso al enigma \\
\hline $\begin{array}{l}\text { 1. Coordenadas de } \\
\text { una figura }\end{array}$ & $\begin{array}{l}\text { "Dicen que la lectura hacía soñar a Don } \\
\text { José. A veces, en mitad de los sueños } \\
\text { despertaba y gritaba:_" (libertad, bès- } \\
\text { ties, fantasma, racisme, y héroe). } \\
\text { La presente pista les guiará hasta la } \\
\text { biblioteca del aula, donde tendrán que } \\
\text { encontrar un libro cuyo título contenga } \\
\text { esta palabra. }\end{array}$ & $\begin{array}{l}\text { Libros de lectura: } \\
\text { - Los hijos de la libertad } \\
\text { - Llibre de les bèsties } \\
\text { - El fantasma de palacio } \\
\text { - El racisme explicat a la meua } \\
\text { filla } \\
\text { - Nunca seré tu héroe }\end{array}$ \\
\hline $\begin{array}{l}\text { 2. Fórmulas } \\
\text { matemáticas }\end{array}$ & $\begin{array}{l}\text { "Sobre vuestras manos acaba de pasar el } \\
\text { enigma 2". } \\
\text { En el mismo libro, en el número de pági- } \\
\text { na del resultado obtenido en el ejercicio } \\
\text { anterior, encontrarán un trozo de papel } \\
\text { en blanco. }\end{array}$ & $\begin{array}{l}\text { Papel en blanco con la fórmula } \\
\text { escrita con tinta invisible. }\end{array}$ \\
\hline $\begin{array}{l}\text { 3. Cálculo del área de } \\
\text { una parte de la figura }\end{array}$ & $\begin{array}{l}\text { "Don José se esforzaba cada mañana en } \\
\text { que sus alumnos empezaran el día con } \\
\text { alegría y motivación". } \\
\text { Cada grupo tendrá una frase diferente } \\
\text { sobre Don José, la cual se relaciona de } \\
\text { forma directa con uno de los } 5 \text { carteles } \\
\text { que se encuentran desapercibidos am- } \\
\text { bientando el aula. }\end{array}$ & $\begin{array}{l}\text { Detrás del cartel, encontrarán un } \\
\text { código QR que les llevará hasta } \\
\text { un blog dónde tendrán publicada } \\
\text { la figura del enigma } 3 \text { y una breve } \\
\text { explicación. }\end{array}$ \\
\hline
\end{tabular}




\begin{tabular}{|c|c|c|}
\hline Enigma & Pista del lugar & Acceso al enigma \\
\hline $\begin{array}{l}\text { 4. Problema } \\
\text { matemático }\end{array}$ & $\begin{array}{l}\text { "Esto no huele nada bien". } \\
\text { Entre los papeles de la papelera del aula, } \\
\text { encontrarán uno de ellos con un texto } \\
\text { escrito del revés. }\end{array}$ & $\begin{array}{l}\text { Folio con el problema escrito del } \\
\text { revés, al cual accederán con la } \\
\text { ayuda de un espejo. }\end{array}$ \\
\hline $\begin{array}{l}\text { 5. Actividad de medi- } \\
\text { ción en el entorno }\end{array}$ & $\begin{array}{l}\text { Palabra o texto en inglés } \\
\text { (perchero, escritorio, radiador, enchufe } \\
\text { y tiza). } \\
\text { En ese lugar encontrarán unos vasos de } \\
\text { plástico que deberán manipular para des- } \\
\text { cubrir la palabra que contiene: corcho. }\end{array}$ & $\begin{array}{l}\text { En el corcho encuentran un co- } \\
\text { rreo electrónico donde se les pide } \\
\text { que envíen un correo electrónico } \\
\text { al game master, solicitando el } \\
\text { siguiente enigma de una forma } \\
\text { original y con un asunto de correo } \\
\text { determinado. }\end{array}$ \\
\hline $\begin{array}{l}\text { 6. Construcción de } \\
\text { una figura a través } \\
\text { del tangram }\end{array}$ & $\begin{array}{l}\text { "Los alumnos y alumnas eran lo más } \\
\text { importante para Don José, no importaba } \\
\text { si sus pupitres eran un desastre, él los } \\
\text { quería tal y como eran". } \\
\text { Escondidos entre los pupitres encontra- } \\
\text { rán una caja bloqueada por un candado. }\end{array}$ & $\begin{array}{l}\text { Para desbloquear el candado, } \\
\text { obtendrán como pista la primera } \\
\text { letra de cada uno de los números } \\
\text { por los que se compone y deberán } \\
\text { probar las diferentes posibilida- } \\
\text { des. Por ejemplo: UTDS (uno, } \\
\text { tres, dos, seis/siete). Una vez } \\
\text { abierto, podrán proceder a montar } \\
\text { el tangram. }\end{array}$ \\
\hline 7. Código morse & $\begin{array}{l}\text { "Mira bien en el interior". } \\
\text { En la misma caja que antes habrá escon- } \\
\text { dido un código QR. }\end{array}$ & $\begin{array}{l}\text { El QR les dará acceso a un audio } \\
\text { de Youtube con un mensaje en có- } \\
\text { digo morse, que deberán escuchar } \\
\text { y traducir con una plantilla del } \\
\text { alfabeto morse camuflada en el } \\
\text { aula. Una vez que hayan traducido } \\
\text { tendrán el lugar donde se encuen- } \\
\text { tra escondido su símbolo final. }\end{array}$ \\
\hline
\end{tabular}

Una vez planteada la narrativa, los enigmas, las pistas y la forma de acceder a ellos, es el momento de analizar el plano del aula para determinar el itinerario a realizar por los grupos y evitar tropiezos o interrupciones entre ellos. La clase, en este caso concreto de escape, no precisará grandes transformaciones de atrezo (puesto que la temática es la desaparición de un profesor del colegio), pero sí deben colocarse algunos detalles para camuflar los objetos y herramientas que servirán para resolver los enigmas. También resultará atractivo colocar algunos elementos decorativos para captar su atención, como cintas policiales en las puertas y ventanas del aula. Como se trata de 
una experiencia con tecnología, todos los alumnos disponen de una tableta y acceso a internet; se pensó en utilizar la herramienta de Google Forms para guiar a los grupos durante el recorrido del escape, creando cuestionarios propios según las necesidades.

En cuanto a la organización del alumnado en grupos, se tuvieron en cuenta diferentes aspectos, valorando tanto las capacidades y el nivel cognitivo como los caracteres y las afinidades entre el alumnado. Se buscó un distintivo para cada grupo, algo sencillo y visual, colgantes de colores (rojo, amarillo, verde, azul y rosa). Y como elemento clave para el desarrollo de la experiencia, la hoja individual de ruta. Se trata de una hoja para cada participante con una tabla en la cual podrá anotar, dentro de cada enigma, cualquier observación, dato o pista que pueda ser relevante. Además, contarán con un espacio donde podrán escribir los cálculos y las operaciones necesarios para resolver los enigmas matemáticos. De esta forma, y puesto que en la escape room el tiempo juega un papel importante, todos los miembros podrán realizar los cálculos al mismo tiempo y contrastar sus resultados, facilitando la interacción, el contraste de ideas y opiniones, y, en definitiva, el aprendizaje.

Para finalizar con este apartado de planificación, la escape room, como cualquier otro juego, precisa de unas reglas que hay que cumplir, las cuales nos garantizarán orden y control.

\subsection{Realización de la de la innovación: observación}

La propuesta se realizó a primera hora de la mañana, uniendo dos sesiones de 45 minutos. El aula se preparó y ambientó según la planificación establecida. Los/as alumnos/as se sentaron formando los grupos que previamente se les había comunicado y se procedió a la entrega de las tarjetas de identificación y las hojas individuales de ruta. Fue en este instante cuando se procedió a la lectura en voz alta de las reglas del juego, así como a la entrega de los comodines y la activación del sonómetro. Del mismo modo, se explicó el funcionamiento de los equipos a través de Google Form. Los cuestionarios se encontraban bloqueados en la primera sección con una clave de acceso que revelaría justo antes de comenzar el juego, de modo que ningún equipo podía leer su primera pista antes de tiempo. 
El inicio del juego vino marcado por la visualización del vídeo de introducción, el cual ponía en contexto al alumnado, quienes a partir de ese momento se convertían en guardias civiles en busca del paradero del docente desaparecido. Y, finalmente, de este modo dio comienzo el juego y la investigación de la desaparición de Don José.

Durante la sucesión del juego, el papel del docente consistió en limitarse a observar y resolver las dudas que iban surgiendo. Para recopilar información durante el juego se utilizaron dos instrumentos: la tabla de resultados del itinerario de cada grupo y un registro de observación.

Finalmente, una vez terminado el juego, se procedió a recoger los materiales y a comentar la experiencia grupalmente. Además, los alumnos rellenaron un cuestionario individual propio para evaluar la experiencia vivida. También se llevó a cabo la entrevista programada con el docente y tutor del grupo, posteriormente a la aplicación de la innovación.

\section{ANÁLISIS DE RESULTAdos}

Uno de los principales objetivos consistía en medir el grado de motivación, de cohesión y de aprendizaje que la escape room podía despertar entre el alumnado. Para ello, de acuerdo con la metodología escogida, pasaremos a analizar uno a uno los tres factores teniendo en cuenta los cuestionarios del alumnado, el registro de observación y la entrevista realizada al tutor del grupo.

\subsection{Análisis del grado de motivación}

Un $86,4 \%$ del alumnado que realizó la experiencia afirma que la temática y narrativa de la escape room ha favorecido que la realización de los problemas y ejercicios planteados sea afrontada con más motivación que en las clases cotidianas. El $100 \%$ del alumnado indica que se ha evadido de su rutina diaria y se ha divertido. De los resultados podemos comprobar que en su mayoría, concretamente un $86,4 \%$, valoran la experiencia en el máximo nivel según la escala likert y el 13,6 \% restante en el nivel 3, de modo que 
podemos afirmar de los datos que la experiencia de escape room ha gustado a todos los/as alumnos/as.

Si analizamos en profundidad el registro de observación con todas las anotaciones realizadas durante la experiencia, observamos grandes diferencias dentro de los grupos. Mientras que los equipos de color azul, amarillo y rosa tienen mayoría de aspas en las emociones positivas y apenas aparecen detectadas expresiones que transmiten emociones negativas, con los equipos de color rojo y verde sucede totalmente al contrario. ¿A qué puede deberse este hecho? Probablemente la respuesta se encuentre en que fueron los dos grupos que más dificultades tuvieron para llegar al final. El rojo, por la dificultad de los enigmas en sí, y el verde, por los conflictos que surgieron dentro del grupo, lo que provocó que fuera el único grupo que no llegara al final de su recorrido. De este modo, observamos dos actitudes claramente opuestas a la hora de hacer frente a las adversidades o problemas: por un lado, aquellos que no se rinden y prueban en múltiples ocasiones hasta dar con la solución, y, por otro, los que con intentarlo una vez tienen suficiente para darse por vencidos, pese a que en ningún momento se llegó a perder la motivación global por el juego.

Respecto a la entrevista con el tutor del grupo, cabe destacar que en la pregunta 1, sobre los motivos por los que optó por emplear este tipo de metodología en el aula, responde rotundamente "sobre todo, porque el grado de motivación es mucho más alto". Además, en la pregunta 3 , sobre los puntos más fuertes de llevar al aula una escape room, responde que "no deja de ser un reto el conseguir escapar de un sitio, encontrar un asesino o una clave, y los retos es una manera de que ellos estén más motivados".

\subsection{Análisis del grado de cohesión grupal}

A la pregunta de si han surgido problemas en el trabajo en equipo, un $68,2 \%$ del alumnado afirma que no han surgido problemas y que han trabajado muy bien en grupo. Pero el 31,8 \% confiesa haberse enfrentado a algún conflicto durante la escape room, aunque, de ellos, el $22,7 \%$ dice que han podido solventar estos conflictos entre ellos sin ayuda del profesor. Por tanto, 
ningún alumno del aula considera que ha sido necesaria la ayuda docente para mediar en los conflictos grupales generados durante la experiencia.

Por otro lado, si analizamos las cuestiones referentes a la cohesión del grupo en la tabla de observación, podemos conocer claramente en qué dos grupos surgieron más conflictos: el equipo rojo y el verde. Se trata justamente de los dos grupos que tuvieron más dificultad para llegar al final; sin embargo, apreciamos una diferencia entre ambos en el ítem 6 "ayuda entre iguales", pues mientras el equipo verde carece prácticamente de cruces en esta casilla, en el equipo rojo sí existió esa cohesión y ayuda por parte de los miembros del equipo para resolver las dificultades surgidas y avanzar. Probablemente sea por esta razón por la que el equipo rojo finalmente sí consiguió llegar hasta el final, no así el equipo verde.

Respecto de la entrevista al tutor, en la pregunta 6 , en la cual se le preguntaba sobre los puntos más fuertes de la propuesta planteada, se respondió lo siguiente: "Sí, la propuesta fue muy arriesgada porque la idea era que todos los alumnos a la vez estuvieran haciendo en la misma sala el escape room. Pero estuvo muy bien dirigida porque cada grupo tenía su ruta y no se chafaban unos a otros. Y conseguir estar dos sesiones sin salir de clase luchando por conseguir el objetivo, pues es un mérito y está muy bien".

Por último, la pregunta número 2, que pretendía profundizar más en el tema, preguntaba por la utilidad de esta metodología para mejorar la cohesión grupal, a lo que el docente respondió: "sí, utilizamos mucho el aprendizaje cooperativo por eso, para que se acostumbren a trabajar en equipo. Como has visto, a veces hay sus problemas, pero en el futuro van a seguir trabajando de esa forma, con lo cual van a tener que empezar a aprender estrategias para convivir entre ellos y aprender a luchar por un objetivo común”.

\subsection{Análisis del grado de aprendizaje}

Atendiendo a los objetivos didácticos de la propuesta, algunos de carácter más conceptual e intelectual, se preguntó la opinión sobre su aprendizaje en el cuestionario. Según lo cual, el $100 \%$ del alumnado contestó que estaba de acuerdo en que la dificultad de los enigmas fue la adecuada. Pero de este $100 \%$, el $40 \%$ lo afirma sin tanta rotundidad (nivel 3). Respecto al tiempo 
destinado a resolver las actividades, hay diferentes opiniones. El 77,3 \% está de acuerdo en que el tiempo es adecuado para resolver los problemas, mientras que el 18,2\% piensa que no es tan adecuado y el 4,5\% está en desacuerdo. De este modo, si contrastamos esta información con las anotaciones del registro de observación, en el ítem "preguntan al profesor para llegar a los enigmas" observamos que sí han existido dificultades; de hecho, se observan más aspas en este apartado que en el número 2, "preguntan al profesor por contenidos", por lo que deducimos que probablemente han tenido más dificultad para acceder a los enigmas a través de las pistas que en la resolución del problema o ejercicio matemático en sí.

Por otro lado, buscando profundizar en el tipo de actividad que les ha resultado más atractiva, la pregunta 8 les pedía que escogieran entre los enigmas que habían implicado el uso de nuevas tecnologías o, por el contrario, los manuales, a lo que ha respondido más de la mitad de la clase, concretamente un $63,6 \%$, que ha disfrutado más con las actividades manuales como el tangram y la construcción de vasos de plástico. Si contrastamos este dato con las respuestas obtenidas en el ítem 3 de la tabla de observaciones, "dificultad en actividades con TIC", observamos cierta coherencia, ya que no hay ninguna cruz marcada; es decir, no se ha observado ninguna dificultad en lo que a las nuevas tecnologías se refiere, por lo que es probable que hayan pasado más desapercibidas debido a su uso cotidiano en el aula.

Además, extraemos de los cuestionarios que al $100 \%$ de los/as alumnos/ as que participaron en la escape room de matemáticas les gustaría repetir este tipo de actividades para aprender los contenidos de otras materias. Y es que, tal y como menciona su tutor en la entrevista, en la pregunta número 3: "no deja de ser un juego que además está de moda y seguramente sus padres paguen por hacerlo, por lo que poder adaptarlo en el colegio y además con los contenidos que van a dar, pues a ellos también les motiva". Además, el tutor afirma en la pregunta 1: "cuanto más motivados están los alumnos, más fácil es que aprendan y estén atentos a lo que hay que hacer".

De este modo, para terminar con este apartado de análisis, comentaremos la pregunta número 9 , la cual iba un poco más allá, puesto que les preguntaba sobre si se veían capaces de realizar ellos mismos la actividad para sus compañeros, a lo que el 81,8 \% consideraba que tal vez sería capaz, y un 13,6 \% 
afirmaba que sí, aunque lo más llamativo de todo es que ningún alumno/a se veía incapaz de hacerlo.

\section{Conclusiones}

No hay nada como poder poner a prueba nuestras ideas y conocimientos para poder observar aquello que funciona y lo que no. En este sentido, el diseño y puesta en práctica de la escape room en el aula ha sido todo un aprendizaje para las personas involucradas en la experiencia, alumnos, docentes e investigadores.

Basándonos en todas las anotaciones y datos registrados durante la investigación, nos atrevemos a afirmar que la experiencia innovadora llevada a cabo en el aula logró motivar a los/as alumnos/as, ya que hemos obtenido resultados muy gratificantes: que la mayoría de la clase ha logrado evadirse y divertirse, que ha encontrado la narrativa atractiva y que estarían dispuestos a repetir la experiencia con contenidos de otras asignaturas.

Del mismo modo, el diseño de la experiencia precisaba un grupo cohesionado para alcanzar el final del juego, y trasmitimos al alumnado la importancia de que los miembros de la clase estuvieran unidos por un objetivo común, ya que todos los grupos de la escape room fueron igual de importantes y necesarios.

Consideramos que la escape room favorece un clima óptimo de aprendizaje, pues el alumnado hace frente a los contenidos de una forma atractiva y disfruta al mismo tiempo que aprende y, en consecuencia, el rendimiento académico que alcanzan es mayor.

Una vez finalizada la investigación y logrados sus objetivos, solo nos queda confirmar o refutar las hipótesis planteadas:

- H.1) La aplicación de la escape room sí ha permitido incrementar la motivación del alumnado.

- H.2) La aplicación de la escape room sí ha permitido trabajar todas las competencias clave de forma integrada.

- H.3) La aplicación de la escape room sí ha permitido generar un aprendizaje activo y basado en el descubrimiento. 
- H.4) La aplicación de la escape room sí ha permitido potenciar la ayuda entre iguales.

- H.5) La aplicación de la escape room sí ha permitido desarrollar estrategias útiles para la resolución de conflictos.

- H.6) La aplicación de la escape room sí ha permitido observar las actitudes, caracteres y roles del alumnado.

Como en todas las investigaciones llevadas al aula, se tienen limitaciones; para poder generalizar conclusiones sería conveniente repetir la misma experiencia de escape room en otros centros educativos, con objeto de comprobar si el éxito de la experiencia se encuentra relacionado con el tipo de alumnado o centro. Del mismo modo, realizar la experiencia con los primeros niveles de educación primaria también nos aportaría datos interesantes para poder analizar el comportamiento de la escape room en alumnos con edades inferiores y con contenidos educativos diferentes.

Consideramos que se trata de una estrategia que aporta al docente muchos datos en relación con las habilidades sociales de su alumnado y que puede ayudarnos a trabajarlo. Por este motivo, consideramos también interesante realizar escape rooms de manera periódica en un centro, por ejemplo, trimestralmente, de modo que se permita analizar la evolución en las actitudes de los/as alumnos/as.

Para finalizar, nos gustaría recuperar el dato del cuestionario del alumnado donde se afirma que todos ellos se ven capaces de plantear una actividad similar a sus compañeros. Este hecho nos hace reflexionar sobre los beneficios que podría tener dejar la escape room en manos del alumnado, aspecto que podría ser una posible futura línea de investigación muy interesante.

\section{BiBLIOGRAFÍA}

ÁreA, M. (2008). La innovación pedagógica con TIC y el desarrollo de las competencias informacionales y digitales. Investigación en la escuela 54, 5-17.

CArr, W. y Kemmis, S. (1986). Teoría crítica de la enseñanza. La investigación-acción en la formación del profesorado. Barcelona: Martínez Roca. 
Cordero, C. (2018). Escape room educativo. Recuperado de: https://www. agorabierta.com/2018/03/escape-room-educativo/.

García, P. A., Gil, J. A., Monteagudo, B., y Navarro, M. (2018). Escapa y aprende: la escape room como estrategia didáctica. España: UNO.

Gil-Quintana, J. y Ortega, R. M. (2018). Gamificación: apostando por una comunicación interactiva y un modelo participativo en educación. Communication papers Media literacy \& Gender Studies 7(14), 9-22.

Gutiérrez, E. (2008). Técnicas e instrumentos de observación de clases y su aplicación en el desarrollo de proyectos de investigación reflexiva en el aula y de autoevaluación del proceso docente. XVIII Congreso internacional de la asociación para la enseñanza de español como lengua extranjera (ASELE). Recuperado de: https://dialnet.unirioja.es/descarga/articulo/ 3189672.pdf.

Latorre, A. (2003). La investigación-acción. Conocer y cambiar la práctica educativa. Barcelona: Graó.

Martínez, A., Poyatos, M. y Fernández, M. (2018). Juegos de fuga para educación: claves para diseñar un break out edu o un escape room para tus alumnos. Recuperado de: http:/www.blogsita.com/wp-content/ uploads/2018/04/break-out-y-escape-room-juegos-de-fuga.pdf.

Mayorga, M. J. (2004). La entrevista cualitativa como técnica de la evaluación de la docencia universitaria. Revista Electrónica de Investigación y Evaluación Educativa 10(1).

Moya, C. (2018). España: líder en usuarios de juegos de escape en Europa. El economista. Recuperado de: https:/www.eleconomista.es/evasion/ caprichos/noticias/9057308/04/18/Espana-lider-en-usuarios-de-juegos -de-escape-en-Europa.html.

NARANJo, M. L. (2009). Motivación: perspectivas teóricas y algunas consideraciones de su importancia en el ámbito educativo. Revista Educación 33(2), 153-170.

Segura-Robles, A. y Parra-GonzÁlez, M. (2019). How to implement active methodologies in Physical Education: Escape Room. ESHPA 3(2), 295306.

Vygotsky, L. S. (1978). El desarrollo de los procesos psicológicos superiores. Barcelona: Grijalbo. 